\title{
A voltage-mode RF DAC for massive MIMO system-on-chip digital transmitters
}

\author{
M. Reza Sadeghifar ${ }^{1,2}$ (1) Håkan Bengtsson ${ }^{1} \cdot$ J. Jacob Wikner ${ }^{2}$
}

Received: 25 February 2019/Revised: 17 June 2019/Accepted: 4 July 2019/Published online: 19 July 2019

(C) The Author(s) 2019

\begin{abstract}
As the number of antenna elements increases in massive multiple-input multiple-output-based radios such as fifth generation mobile technology $(5 \mathrm{G})$, designing true multi-band base-station transmitter, with efficient physical size, power consumption and cost in emerging cellular frequency bands up to $10 \mathrm{GHz}$, is becoming a challenge. This demands a hard integration of radio components, particularly the radio's digital application-specific integrated circuits (ASIC) with high performance multi-band data converters. In this work, a novel radio frequency digital-to-analog converter (RF DAC) solution is presented, that is also capable of monolithic integration into today's digital ASIC due to its digital-in-nature architecture. A voltage-mode conversion method is used as output stage, and configurable mixing logic is employed in the data path to create a higher frequency lobe and utilize the output signal in the first or the second Nyquist zone. This 12-bit RF DAC is designed in a $22 \mathrm{~nm}$ FDSOI CMOS process, and shows excellent linearity performance for output frequencies up to $10 \mathrm{GHz}$, with no calibration and no trimming techniques. The achieved linearity performance is able to fulfill the high requirements of 5G base-station transmitters. Extensive Monte-Carlo analysis is performed to demonstrate the performance reliability over mismatch and process variation in the chosen technology.
\end{abstract}

Keywords Radio frequency digital-to-analog converter - RF-sampling DAC · RF DAC · Digital transmitter · Digital-to-RF converter $\cdot$ Software-defined radio $\cdot$ Massive MIMO $\cdot 5 \mathrm{G}$

\section{Introduction}

Massive multiple-input, multiple-output (MIMO) wireless technology is a key in the fifth-generation (5G) mobile telephony standard to achieve higher information transfer capacity by employing spatial multiplexing and beamforming techniques. Information transfer capacity can be improved by increasing the bandwidth and by increasing the number of parallel data streams via spatial multiplexing and beamforming [9]. The massive MIMO-based 5G

\footnotetext{
M. Reza Sadeghifar

reza.sadeghifar@ericsson.com

Håkan Bengtsson

hakan.bengtsson@ericsson.com

J. Jacob Wikner

jacob.wikner@liu.se

Ericsson AB, Radio Systems, Stockholm, Sweden

2 Department of Electrical Engineering, Linköping University, 58183 Linköping, Sweden
}

standard is exploiting multi-path propagation and spatial multiplexing to send and receive multiple data streams over the same radio channel by beamforming technique and hence increasing the system capacity and spectral efficiency [9].

As the number of antenna elements increases in massive MIMO radios, designing efficient radio base-stations covering multiple bands in existing, unlicensed, or emerging frequency bands up to $10 \mathrm{GHz}$ [6], with efficient size, cost, and power consumption is becoming a challenge. This demands a hard integration of the radio components and specifically the digital ASIC with high-performance data converters. For instance, in downlink, monolithic integration of RF DAC and digital ASIC into "one" digital radio System-On-Chip (SoC) results in reduced power consumption as well as a considerable footprint reduction and thus the size of the radios. To achieve this, high performance data converters to fulfill the wireless infrastructure requirements with very high output bandwidth to cover 
multiple frequency bands, and in the same time, compatible with digital integration are highly demanded.

Figure 1 shows a block diagram of a possible implementation of a transmitter for 5G. There are multiple antenna elements (for instance 64), in order to form highly focused beams. Each branch mainly consists of a digital signal processing (DSP) block, RF DAC, power amplifier (PA) and band filter. Each branch delivers the pre-processed data stream with high power and proper phase difference to each antenna element. Depending on if the beamforming is digital, analog or hybrid, the phase adjustment between the branches are performed in digital, RF chain or both, respectively [23]. In Fig. 1(a), digital beamforming architecture is assumed which requires the same number of RF DACs as the number of antenna elements. In each branch, the baseband in-phase (I) and quadrature-phase $(\mathrm{Q})$ data are fed to digital up-conversion block (DUC) where the data is interpolated and up-sampled to the RF DAC update rate, and also digital IQ modulation is performed. The numerically controlled oscillator (NCO) provides $0^{\circ}$ and $90^{\circ}$ shifted phase LO to the digital IQ modulator. There are advantages with digital IQ modulation, comparing to analog IQ modulation, such as, IQ imbalance can be digitally canceled and there is no LO feedthrough that falls into the carrier bandwidth. The realvalued digital signal is then fed to the RF DAC to be represented by an analog signal. The RF DAC directly

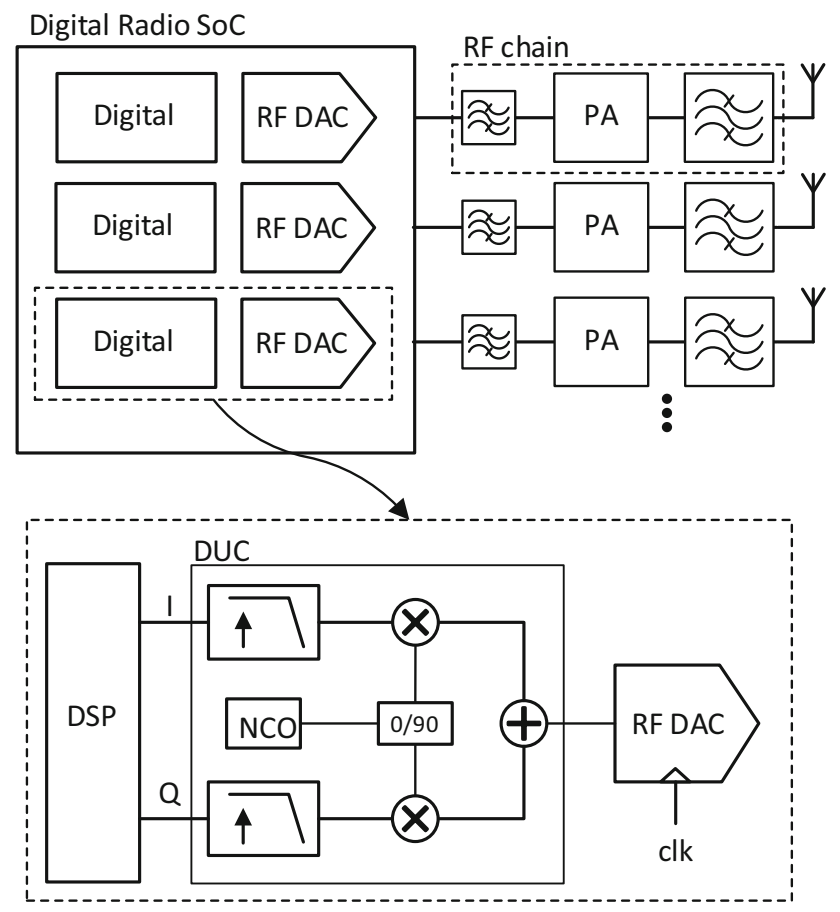

Fig. 1 Block diagram representation of a massive MIMO-based transmitter with monolithic integration of data converters into the digital Radio System-On-Chip synthesizes the signal in the desired RF frequency and there is no need for another frequency translation step. The sample frequency is provided by a high-performance phase-locked loop (PLL) to the RF DAC. If the RF DAC is used in mixing mode configuration, the LO frequency does not have to be higher than the output RF signal, which is a big advantage for this method, would otherwise require more than double the output frequency in conventional Nyquist DACs.

The rest of this paper is formatted as follows: In Sect. 2, digital transmitters employing RF DACs in literature are reviewed, in Sect. 3, the proposed RF DAC-based digital transmitter is described. Details of circuit implementation and simulation analysis and impact of non-idealities such as device mismatch induced errors are analyzed and presented in Sect. 4, and concluding remarks in Sect. 5.

\section{Digital transmitter}

In conventional analog transmitters with one to four antennas (as in 3G and 4G), a high performance DAC, in terms of linearity and noise spectral density, and an analog IQ modulator have been used. The DAC often is operating in the first Nyquist zone and the DAC output signal is in the sub-GHz range. The IQ modulator translates the analog signal to the desired RF band. As the number of antenna elements grows, for instance in LTE-A, to up to eight elements per radio, the integration of the baseband DAC and analog IQ modulator was more efficient in terms of overall radio size and cost $[13,14,21]$. In massive MIMO however, the number of antenna elements increases dramatically, and a digital transmitter with an RF DAC solution is more beneficial as there is no analog mixer limitation and therefore simultaneous multi-band operation becomes easier. In a digital transmitter with digital IQ modulator, the RF DAC converts digital data directly to RF signal which goes to the power amplifier (PA) and the antenna, simplifying the overall radio design and results in smaller area and cost. If in a radio system, the digital ASIC and the digital transmitter (including digital IQ modulator and the RF DAC) are implemented in separate chipsets, multigiga-bit serial data link (such as JESD204B) between the digital ASIC and the digital transmitter, requires a huge power consumption, both in the digital ASIC as the sender of high-speed serial data and the RF DAC as the receiver. If the RF DAC is integrated into digital ASIC (on the same die), it will be possible to save power and area by eliminating the serial link transceivers. The RF DAC can potentially fit in the same area as of the high-speed serial data link sender in digital ASIC, which implies no additional area is needed for monolithic integration of the RF DAC in digital ASIC [5]. 


\subsection{RF-sampling DACs}

RF DAC or RF-sampling DAC, in this context, refers to DACs for which the sample rate is high and the DAC output is in RF domain $[2,3,15,18]$. Depending on the architecture and implementation choices, the RF DAC can utilize first, second or even higher Nyquist zones to synthesize the signal at the desired RF frequency. In a zeroorder hold RF DAC, the analog signal is reconstructed by means of pulse-amplitude modulation (PAM) of the digital input data using a rectangular pulse with duration of sampling period $T_{s}=1 / f_{s}$, and the frequency response is a sinc-weighted function with zeros at multiples of the sample frequency $f_{s}$. The sample rate in this case must be very high, for instance, more than $20 \mathrm{GHz}$ to cover up to $10 \mathrm{GHz}$ RF output within the first Nyquist zone. Higher Nyquist images of the DAC in this structure will have 10 to $20 \mathrm{~dB}$ less power and hence degraded dynamic range and dynamic linearity.

In mixing RF DACs, however, the PAM signal can be an oscillating pulse, for which the amplitude is modulated with the level of the input digital code which gives rise to the higher Nyquist images energy. The frequency response of the RF DAC will have high energy lobe at $f_{s}$ or a multiple of $f_{s}$ depending on the oscillating pulse duration. The higher Nyquist images of the digital data at $f_{s}$ (or a multiple of $f_{s}$ ), will be the RF output signal of the RF DAC $[5,7,12,19]$.

\section{Proposed RF DAC-based digital transmitter}

In this work, a 12-bit resolution RF DAC solution for a digital radio transmitter is presented. The digital data is processed in the digital blocks and the sample rate is increased to the update rate of the RF DAC.

In general two approaches exist for implementing a mixing RF DAC depending on how the mixing operation in each cell of the DAC is performed, as discussed in [19]: at the data path as "mixing logic" or modulating the tail current as "series mixing" and therefore various oscillating PAM signals have been used for RF DAC implementation such as continues sinusoidal [7, 12], discrete oscillating PAM [20], or a bipolar rectangular [5, 19]. To implement the RF DAC in a process that is compatible with co-integration by digital ASIC, mixing logic approach is preferred due to the voltage headroom limitations and also the "digital-in-nature" characteristic of this method, so that the RF DAC actually benefits from process scaling.

\subsection{RF DAC architecture}

A top-level functional block diagram of the proposed RF DAC is shown in Fig. 2 which contains digital processing block for segmentation and decoding and a number of weighted unit conversion cells that are driven by the decoded input bits. Each conversion cell is constructed of data capturing and mixing logic, and voltage-mode conversion block. The positive and negative output of all conversion cells are combined and terminated in a transformer with differential $100 \Omega$ load. The transformer at the load will help isolating the output voltage swing from the internal RF DAC nodes, as well as impedance matching if required. It will also help for common-mode isolation and required voltage headroom. There is a trade-off here between selecting on-chip or off-chip transformer. An offchip transformer requires a large footprint and bond wire inductance can be challenging at higher frequencies. An on-chip transformer comes at the cost of larger chip area and larger power dissipation for the RF DAC output stage.

To implement the mixing logic function, the PAM signal is selected to be a bipolar pulse toggling between amplitudes $+A$ and $-A$ (Fig. 3b), where $A$ is the level corresponding to the input digital code. This PAM signal can be generated by applying an exclusive NOR logic gate (XNOR) to the clock and the input bit as shown in Fig. 3(a and $b)$.

\subsection{RF DAC output stage in voltage-mode}

Traditionally, current-steering DACs have been the choice for high speed application mainly because of the driving capability of the current sources in high speed operation and to avoid having buffer at the output stage of the DAC. Switching time variation which is input code-dependent is one source of non-linearity. Another one, which is the main limitations of dynamic performance in current steering DACs, is the finite output impedance of the unit current cell. This makes the DAC output impedance input codedependent, and causes non-linearity $[10,16]$. There are some techniques to overcome this limitation to some extent, for instance, by using bleeding current sources $[5,10]$, still the achievable linearity for sub-10 GHz operation is very limited.

In the proposed RF DAC solution, Voltage-mode (VM) conversion is used as the output stage of the RF DAC, as shown in Fig. 2 and each data bit, after passing through the mixing logic, is applied to the switches $d$ and $d_{N}$. These switches are implemented by means of an inverter connecting the positive and negative terminals of the output to either $V_{\text {ref+ }}$ or $V_{\text {ref- }}$ through the unit element resistors $\left(R_{u}\right)$. Due to high speed switching capability of the CMOS 
Fig. 2 Top-level functional block diagram of the proposed RF DAC for a digital RF transmitter with an off-chip Balun

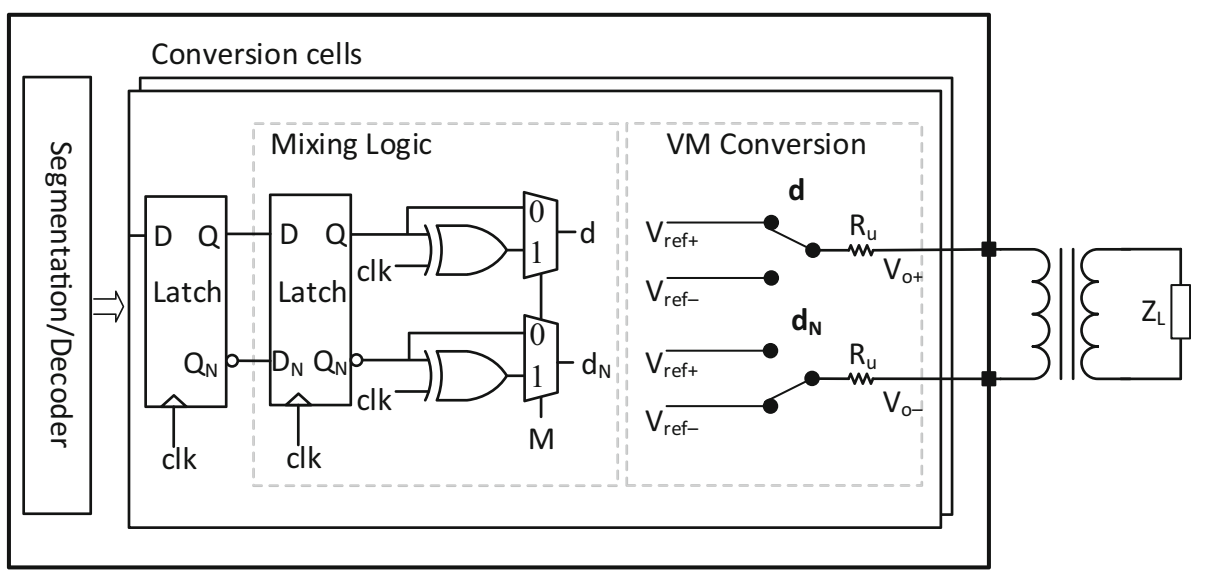

Fig. 3 a Mixing logic operation by XNOR-gating the input bit and the clock, $\mathbf{b}$ bipolar pulse amplitude modulation (PAM) to generate the RF DAC frequency response, and $\mathbf{c}$ simplified equivalent circuit at each clock phase

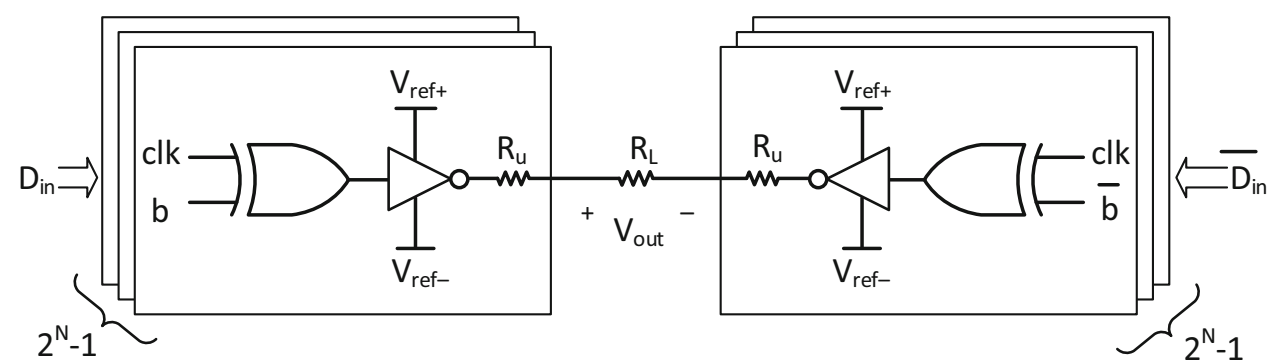

(a)

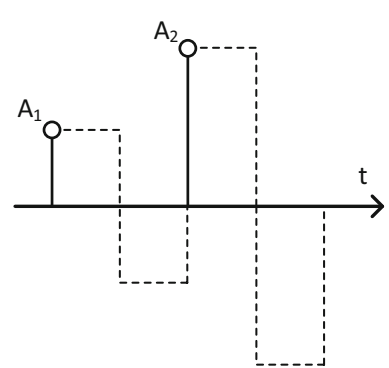

(b)

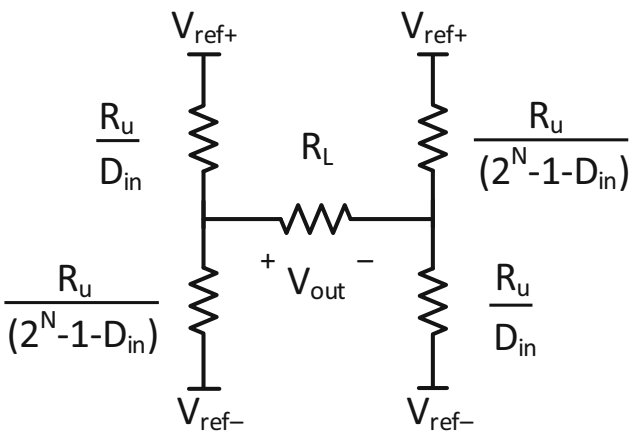

(c) transistor in this technology, very high speed RF DAC operation will be possible. This switching speed will benefit from process scaling and will become even faster in advanced nodes.

\subsubsection{Transfer function}

The voltage-mode conversion and mixing logic is shown in Fig. 3(a). At each clock phase the output stage will become a resistor network as shown in Fig. 3(c), and the steadystate transfer function, output voltage versus input code, can be calculated investigating the half-differential Thevenin equivalent as:

$$
\begin{aligned}
V_{t h} & =\frac{D_{i n}}{2^{N}-1} \cdot\left(V_{r e f+}-V_{r e f-}\right), \\
R_{t h} & =\frac{R_{u}}{2^{N}-1} .
\end{aligned}
$$

The differential output voltage is then derived as

$$
V_{\text {out }}=\frac{2 D_{\text {in }}-\left(2^{N}-1\right)}{\left(2^{N}-1\right)+2 R_{u} / R_{L}} \cdot\left(V_{\text {ref }+}-V_{\text {ref- }}\right) \text {, }
$$

where $D_{\text {in }}$, is the input code, $R_{L}$ is differential load virtually seen by the RF DAC output and $R_{u}$ is the unit element resistor. In this architecture, the impedance seen from the output of the RF DAC, is always constant regardless of what input code is applied. That is, all the $R_{u}$ resistors are either connected to positive or negative reference voltage 
and hence the total output impedance is always constant and it does not contribute to the linearity degradation. This can be also observed from (2) where the output voltage is a linear function of the input code and there is no codedependent load variation which was a limiting factor in current steering DACs.

However, switching time variation will still impact the non-linearity performance. The "on" resistance of the switches is negligible comparing to the individual unit element resistors in series $\left(R_{u}=10 \mathrm{k} \Omega\right)$, and therefore is ignored in (2) for simplicity. Unit element resistors are implemented in $\mathrm{N}+$ Polysilicon material in this $22 \mathrm{~nm}$ FDSOI CMOS process. Each $R_{u}$ has a width of $360 \mathrm{~nm}$ and a length of $7.6 \mu \mathrm{m}$ and resistance of $10 \mathrm{k} \Omega$. If resistance value of $R_{u}$ is too small, and once all in parallel, comparable to the "on" resistance of PMOS and NMOS switches, there will be code-dependent load variations due to the differences of PMOS and NMOS transistor "on" resistance. The optimum unit element resistor is found by sweeping its value and the simulation shows that smaller values cause more non-linearity and larger values limit the achievable speed due to more parasitic capacitors and hence $R_{u}=10 \mathrm{k} \Omega$ seems to be a good compromise. The overall RF DAC core area is dominated by the unit element resistors. In 12 bit RF DAC, there are 4095 unit elements and each $R_{u}$ has an area of $2.74 \mu \mathrm{m}^{2}$, in total becomes $0.00112 \mathrm{~mm}^{2}$. Although this is only the area needed for resistors and not the complete design, it gives a good approximation of the required area and indicates that the proposed RF DAC area is very competitive, when compared to the state of the art RF DACs [5, 13, 14]. The NMOS and PMOS switches in each unit element are minimum sized $(\mathrm{W}=300 \mathrm{~nm}, \mathrm{~L}=20 \mathrm{~nm})$ with $\mathrm{m}$-factor of five (transistors $M_{1-4}$ in Fig. 5).

\subsubsection{Unit Element Resistors Mismatch and RF DAC Segmentation Scheme}

Process and mismatch variation within RF DAC cells will cause errors in timing and amplitude accuracy in multi-bit RF DAC [11]. The unit element mismatch error can be modeled as an additive random variable, where the additive random variable's standard deviation depends on the desired value. According to Pelgrom's model [8, 17], the standard deviation of mismatch is inversely proportional to the area and hence more resistors in parallel decreases the mismatch.

In order to characterize the resistor mismatch behavior in the selected process, $22 \mathrm{~nm}$ FDSOI CMOS, a single nominal $10 \mathrm{k} \Omega$ Poly resistor with size of $\mathrm{W}=0.36 \mu \mathrm{m}$ and $\mathrm{L}=7.6 \mu \mathrm{m}$ is simulated with 1000 Monte-Carlo samples and the simulated resistance value is observed. As discussed before, the unit element resistor value is selected as $R_{u}=10 \mathrm{k} \Omega$ given the trade-off between the output switching speed due to parasitic capacitance and being sufficiently large value compared to the "on" resistance of the switches.

The simulation is run in three different mode, first only mismatch, second only process and third with both mismatch and process variation and the one-sigma standard deviation values and mean values are listed in Table 1, where $\epsilon$ is a random error that represents the fluctuation around the nominal value of the resistor in $\Omega$, and $\mu$ is mean value in $\Omega$ and $\sigma$ is standard deviation of the resistor in percentage relative to the mean value.

In this work, the unit element conversion cells are weighted according to the segmented bits as shown in Fig. 4. The first six LSBs $(L=6)$ are fed to binary weighted unit element conversion cells, and the remaining MSBs (M $=6$ ) are decoded into 63 unary bits, and corresponding cells are weighted as $2^{L}=64$.

The same Monte-Carlo simulation is also run for the MSB, i.e., 64 resistors of $10 \mathrm{k} \Omega$ in parallel, and the result is shown in Table 2.

As expected from Pelgrom's model [8, 17], the mismatch variation (within die) is scaled with the square root of area, i.e., 64 parallel resistors have 64 times more area and hence the corresponding sigma is 8 times smaller $(0.16 \%=1.24 \% / \sqrt{64})$, while the process variation (dieto-die) does not scale with area. With this segmentation scheme (M=6, $\mathrm{L}=6)$ the largest error which is on the LSB $R_{u}(124 \Omega)$ will be less than nominal value of the MSBs $(156.25 \Omega)$.

Although for the dynamic performance it is the mismatch variation only which is important, to ensure that it covers the worse cases, the simulations in the following sections are run with both mismatch and process variation indicating that the simulation result is conservative.

\section{Implementation and simulation analysis}

The proposed RF DAC solution is implemented in $22 \mathrm{~nm}$ fully depleted silicon-on-insulator (FDSOI) CMOS technology and simulated with the spectre simulator engine. The transistor-level schematic diagram of a unit conversion

Table 1 One unit element resistor $R_{u}$ with nominal value of $10 \mathrm{k} \Omega$

\begin{tabular}{llll}
\hline & Mismatch & Process & Mismatch and process \\
\hline$\epsilon, \Omega$ & 124.1 & 699.1 & 665 \\
$\mu, \mathrm{k} \Omega$ & 10.01 & 10.03 & 10.03 \\
$\sigma, \%$ & 1.24 & 6.97 & 6.6 \\
\hline
\end{tabular}




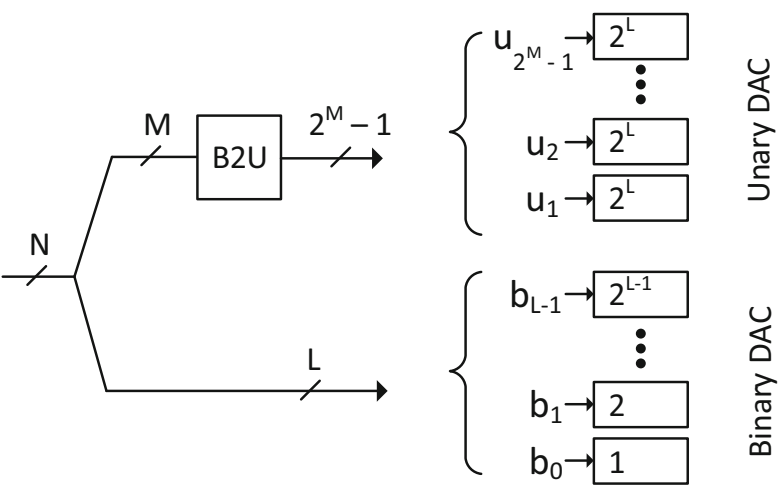

bit split and unary decode

Fig. 4 Segmentation scheme and unit conversion cells weighting

Table 264 unit element resistors $R_{u}$ in parallel with nominal value of $10 k / 64=156 \Omega$

\begin{tabular}{llll}
\hline & Mismatch & Process & Mismatch and process \\
\hline$\epsilon, \Omega$ & 0.249 & 10.92 & 10.38 \\
$\mu, \Omega$ & 156.3 & 156.7 & 156.7 \\
$\sigma, \%$ & 0.16 & 6.97 & 6.6 \\
\hline
\end{tabular}

cell, consisting of data capturing latch, re-timing latch and mixing logic, and voltage-mode conversion stage, is shown in Fig. 5. Signal $b$ is the input bit to each cell using a differential clock signal $c l k_{p}$ and $c l k_{n}$ with 5-ps rise- and fall times. Signal $M$ is selecting mixing or base-band mode

operation of the RF DAC. $V_{r e f+}$ and $V_{r e f-}$, are the reference voltages to the output stage. $V_{r e f-}$ is $0 \mathrm{~V}$ and connected to ground and $V_{\text {ref+ }}$ is supplied from a $0.8 \mathrm{~V}$ stable power supply with minimum disturbances and large decoupling capacitors.

\subsection{Impact of mismatch on performance}

Process and mismatch variation will cause errors in timing and amplitude accuracy of the RF DAC and can degrade the performance. Amplitude error is dominated by the unit element resistors mismatch. To mitigate the mismatch error, potentially, a dynamic element matching (DEM) scheme can be adopted to average out the spurious, with the penalty on the increased noise floor due to randomization. The skew variation between different clocking routes to RF DAC unit elements are caused by the device mismatch and can also be averaged-out by randomization. The timing jitter (clock phase noise), however, changes stochastically at every sampling edge and for all unit elements, and is not mitigated by randomization technique. The simulation result in this work is without the use of dynamic element matching since the noise spectral density (NSD) requirement of the RF DAC in 5G base-station application is very tough and any increase in the noise level is undesired. It should also be mentioned that the RF DAC noise specification is affected by the PLL phase noise.

In order to analyze the impact of mismatch on linearity performance, extensive Monte-Carlo simulation is performed on the proposed RFDAC circuit and the result is presented in Sect. 4.4.
Fig. 5 Complete transistor-level schematic diagram of one conversion unit element cell, including the latches, mixing logic and voltage-mode (VM) conversion

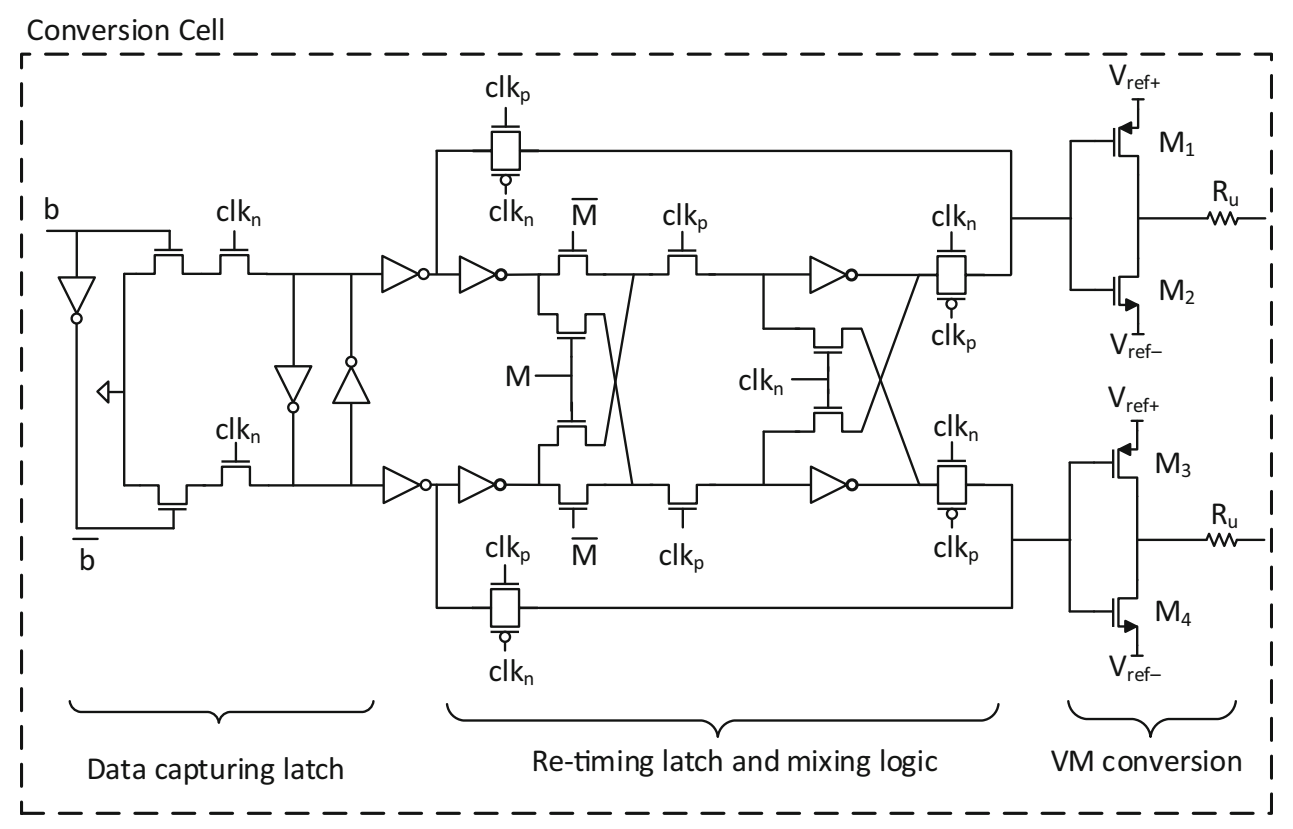




\subsection{Impact of resistor thermal noise}

There are $2^{N}-1$ resistors in parallel at each clock phase, connected to the reference voltages. The uncorrelated noise power is added from each resistor and the total noise power will be

$\overline{I_{n, t o t}^{2}}=\overline{I_{n, 1}^{2}}+\overline{I_{n, 2}^{2}}+\cdots=\frac{4 k T}{\left(\frac{R_{u}}{2^{N}-1}\right)}$,

$\overline{V_{n, t o t}^{2}}=\overline{I_{n, t o t}^{2}}\left(\frac{R_{u}}{2^{N}-1}\right)^{2}=4 k T\left(\frac{R_{u}}{2^{N}-1}\right)$,

which implies that the thermal noise power of all resistors in parallel, is divided by the total number of unit elements and will be $2^{N}-1$ times smaller than single $R_{u}$.

\subsection{Simulation setup}

Simulation results confirms the discussion on linearity performance in the previous sections. A test case with a $10 \mathrm{GHz}$ clock frequency $\left(f_{s}\right)$ and $R F_{\text {out }}$ up to $10 \mathrm{GHz}$, is set up and simulated. Differential load of $100 \Omega$, with $300 \mathrm{fF}$ capacitive load, is used. In a complete system, the digital input base-band frequency is translated to the desired RF frequency, from DC to Nyquist frequency $\left(f_{s} / 2\right)$, by means of digital up-converter (DUC). Since implementing the $\mathrm{NCO}$ and DUC, is out of scope of this work, the input signal to the RF DAC is directly generated with proper frequency in the simulator. For RF signals within first Nyquist zone, the base-band mode of operation of the RF DAC is selected and for RF signals at second Nyquist zone, the RF DAC is configured to operate in mixing mode to get higher power level from the output signal.

\subsection{Monte-Carlo analysis}

As discussed before mismatch and process variation will impact the RF DAC performance. Particularly unit element resistors mismatch will result in degraded linearity performance. In order to analyze the unit element resistors mismatch impact, 100-point Monte-Carlo simulation is run and Fast Fourier transform (FFT) is performed on the output transient signal for each Monte-Carlo run. Third and fifth order intermodulation distortions (IM3 and IM5) are measured by two-tone test, each tone at $-6 \mathrm{dBFS}$, and $15 \mathrm{MHz}$ frequency spacing between tones. Figure 6 shows the worse-case IM3 and average IM3 performance and total output RF power in six different output frequencies. The worse-case IM3 better than $70 \mathrm{dBc}$ for $f_{\text {out }}$ up to $10 \mathrm{GHz}$ is achieved with output RF power $>0 \mathrm{dBm}$. The IM3 and IM5 distribution of 100 Monte-Carlo simulation points is shown in Fig. 7 for three different output frequencies. The output spectrum for worse-case mismatch

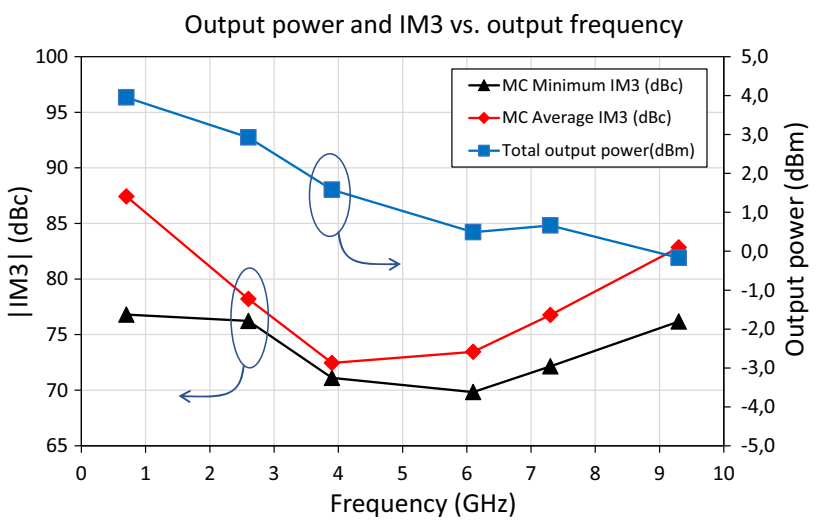

Fig. $6 P_{\text {out }}$ and IM3 performance in 100 Monte-Carlo points, for output signals from DC to $f_{s}$. The sample frequency is at $10 \mathrm{Gsps}$

corner, at $9.3 \mathrm{GHz}$ output $\mathrm{RF}$ frequency is shown in Fig. 8 and demonstrates IM3 of $76 \mathrm{dBc}$ while maintains excellent spurious free dynamic range (SFDR) in the Nyquist bandwidth of $5 \mathrm{GHz}$.

\subsection{Parasitic capacitance of routing unit elements}

To avoid the asymmetry between unit elements caused by the layout effects, the routing strategy should be selected in layout such that the parasitic capacitance of the metal wires connecting the unit elements to the differential output terminals are as similar as possible. One approach to keep the symmetry is to select a tree structure for output routing [22].

To consider this parasitics impact in our analysis, an experiment is done in which extra capacitance instances are added in the schematic of each cell, after each unit element resistor to account for the routing parasitic capacitance. This parasitic capacitance is swept up to $20 \mathrm{fF}$ and the circuit is simulated in one of the worse Monte Carlo corners $\left(f_{\text {out }}=2.6 \mathrm{GHz}\right)$ to observe the effect. One should note that $20 \mathrm{fF}$ at each cell for parasitic capacitance is a very conservative choice and the parasitics are typically smaller than this value. In the worse case, IM3 is degraded with approximately $2 \mathrm{~dB}$ considering the parasitic capacitance of $20 \mathrm{fF}$. As expected going from schematic to layout the performance will be changed, however as simulations show, this performance degradation is within the expected range.

\section{Comparison and conclusion}

An RF DAC solution with capability to be monotonically integrated into digital system-on-chip, and complying with the area and power consumption constrains of massive 
Fig. 7 IM3 and IM5 distribution of 100 Monte-Carlo points, for three different $f_{\text {out }}: 2.6,6.1$ and $9.3 \mathrm{GHz}$. The sample frequency is at $10 \mathrm{Gsps}$
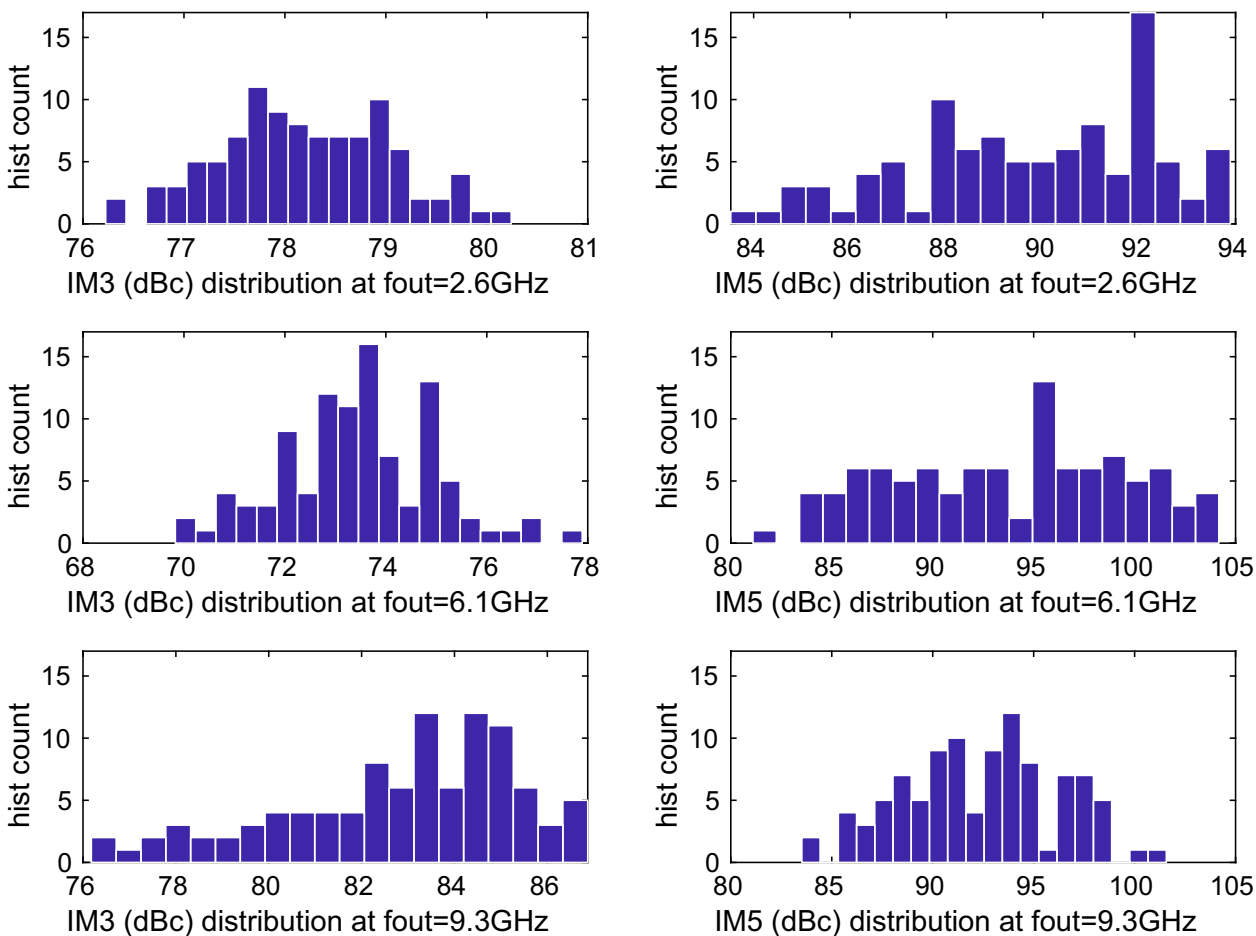

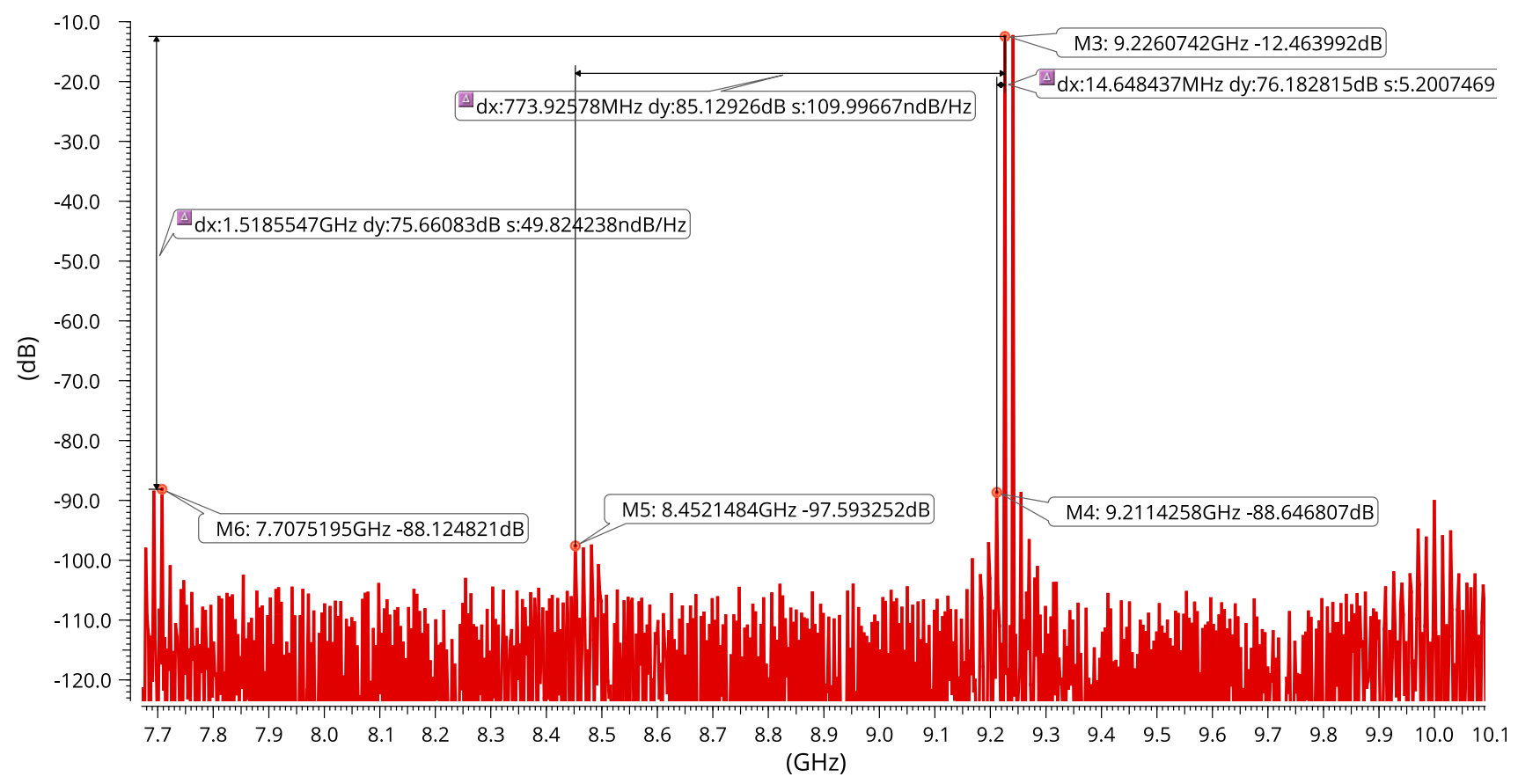

Fig. 8 Two-tone test spectrum for the worst-case mismatch corner at $f_{\text {out }}=9.3 \mathrm{GHz}$, demonstrating an IM3 of $>76 \mathrm{dBc}$. The sample frequency is at $10 \mathrm{GSps}$. Folded second and third harmonic distortion

MIMO-based 5G transmitters in cellular communication, is described in this paper. A proof-of-concept of this architecture were designed and implemented by a 12-bit segmented RF DAC. components are also marked in the plot and shows $>75 \mathrm{dBc}$ SFDR within the Nyquist band

The impact of the major circuit imperfections is studied analytically in this work and it is shown in principle with the aid of extensive Monte-Carlo simulations, that in worse-case corners, the performance is still able to fulfill the upcoming $5 \mathrm{G}$ standard requirements. Since the 
proposed RF DAC is very compact in size, it can replace high-speed serial data link block in a digital ASIC meaning that basically the monolithic integration of the RF DAC comes with no die-area increase for the digital ASIC in fully digital beamforming $5 \mathrm{G}$ architectures.

The proposed work is compared against some of the recent state-of-the-art RF DACs $[1,4,11]$. These RF DACs are implemented in current-steering architecture. There are non-idealities associated with the current source implementation as well. Finite output impedance of the unit element current sources causes harmonic distortion and mismatch between the unit element current sources causes timing and amplitude inaccuracy and hence harmonic distortion. To mitigate the finite output impedance of current-steering architecture, works in $[1,4,11]$ utilize the bleeding current source technique proposed in [10], making the output impedance of the DAC independent of the input code. Moreover in a current-steering structure the mismatch can happen between unit current sources and also between the switches. The former causes amplitude error and the latter will cause timing error between the samples. These errors appear as spurious emission in spectrum of the DAC and hence limiting the dynamic linearity. The work in [11] utilizes calibration technique to mitigate the amplitude errors and utilizes Dynamic Element Matching (DEM) technique to mitigate the timing errors. In this way the randomization via DEM technique alleviates spurious problem without increasing the noise spectral density.

Using the aforementioned techniques, the work in [11] achieves IM3 better than $70 \mathrm{dBc}$ up to $4.5 \mathrm{GHz}$ output frequency and the work in [4] achieved IM3 better than $65 \mathrm{dBc}$ up to $6.8 \mathrm{GHz}$ output frequency, both in $16 \mathrm{~nm}$ CMOS technology. The proposed solution in this work has shown in simulations to be able to achieve better than $70 \mathrm{dBc}$ IM3 in output frequencies up to $10 \mathrm{GHz}$ in $22 \mathrm{~nm}$ CMOS technology without any calibration, trimming or compensation method.

Acknowledgements Open access funding provided by Linköping University.

Open Access This article is distributed under the terms of the Creative Commons Attribution 4.0 International License (http://creative commons.org/licenses/by/4.0/), which permits unrestricted use, distribution, and reproduction in any medium, provided you give appropriate credit to the original author(s) and the source, provide a link to the Creative Commons license, and indicate if changes were made.

\section{References}

1. Bechthum, E., Radulov, G., Briaire, J., Geelen, G., van Roermund, A . (2015). A 5.3ghz 16b 1.75gs/s wideband rf mixing-dac achieving imd $<-82 \mathrm{dbc}$ up to $1.9 \mathrm{ghz}$. In 2015 IEEE international solid-state circuits conference-(ISSCC) digest of technical papers, (pp. 1-3). https://doi.org/10.1109/ISSCC.2015.7062980.

2. Bechthum, E., Radulov, G. I., Briaire, J., Geelen, G. J. G. M., \& van Roermund, A. H. M. (2016). A wideband RF mixing-DAC achieving IMD <-82 dBc up to $1.9 \mathrm{GHz}$. IEEE Journal of SolidState Circuits, 51(6), 1374-1384. https://doi.org/10.1109/JSSC. 2016.2543703.

3. Engel, G., Clara, M., Zhu, H., Wilkins, P. (2015). A 16-bit 10Gsps current steering RF DAC in 65nm CMOS achieving 65 $\mathrm{dBc}$ ACLR multi-carrier performance at $4.5 \mathrm{GHz}$ Fout. In 2015 Symposium on VLSI circuits (VLSI circuits) (pp. C166-C167). https://doi.org/10.1109/VLSIC.2015.7231252.

4. Erdmann, C., Cullen, E., Brouard, D., Pelliconi, R., Verbruggen, B., Mcgrath, J., Collins, D., Torre, M.D.L., Gay, P., Lynch, P., Lim, P., Collins, A., Farley, B. (2017). A 330 mW 14b 6.8 GS/s dual-mode RF DAC in $16 \mathrm{~nm}$ FinFET achieving $-70.8 \mathrm{dBc}$ ACPR in a $20 \mathrm{MHz}$ channel at $5.2 \mathrm{GHz}$. In 2017 IEEE international solid-state circuits conference (ISSCC) (pp. 280-281). https://doi. org/10.1109/ISSCC.2017.7870370.

5. Farley, B., Erdmann, C., Vaz, B., McGrath, J., Cullen, E., Verbruggen, B., Pelliconi, R., Breathnach, D., Lim, P., Boumaalif, A., Lynch, P., Mesadri, C., Melinn, D., Yap, K.P., Madden, L. (2017) A programmable RFSoC in $16 \mathrm{~nm}$ FinFET technology for wideband communications. In 2017 IEEE Asian solid-state circuits conference (A-SSCC) (pp. 1-4). https://doi.org/10.1109/ ASSCC.2017.8240201.

6. ITU. (2015). Technical feasibility of IMT in bands above $6 \mathrm{GHz}$. https://www.itu.int/pub/R-REP-M.2376. Accessed 1 June 2019.

7. Jerng, A., \& Sodini, C. (2007). A wideband $\Sigma \Delta$ digital-RF modulator for high data rate transmitters. IEEE Journal of SolidState Circuits, 42(8), 1710-1722. https://doi.org/10.1109/JSSC. 2007.900255.

8. Kinget, P. R. (2005). Device mismatch and tradeoffs in the design of analog circuits. IEEE Journal of Solid-State Circuits, 40(6), 1212-1224. https://doi.org/10.1109/JSSC.2005.848021.

9. Larsson, E. G., Edfors, O., Tufvesson, F., \& Marzetta, T. L. (2014). Massive MIMO for next generation wireless systems. IEEE Communications Magazine, 52(2), 186-195. https://doi. org/10.1109/MCOM.2014.6736761.

10. Lin, C. H., van der Goes, F. M. L., Westra, J. R., Mulder, J., Lin, Y., Arslan, E., et al. (2009). A 12 bit 2.9 GS/s DAC with IM3<60 $\mathrm{dBc}$ beyond $1 \mathrm{GHz}$ in $65 \mathrm{~nm}$ CMOS. IEEE Journal of Solid-State Circuits, 44(12), 3285-3293. https://doi.org/10.1109/JSSC.2009. 2032624.

11. Lin, C.H., Wong, K.L.J., Kim, T.Y., Xie, G.R., Major, D., Unruh, G., Dommaraju, S.R., Eberhart, H., Venes, A. (2018). A 16b 6GS/S nyquist DAC with IMD $<-90 \mathrm{dBc}$ up to $1.9 \mathrm{GHz}$ in $16 \mathrm{~nm}$ CMOS. In 2018 IEEE international solid-state circuits conference-(ISSCC) (pp. 360-362). https://doi.org/10.1109/ISSCC. 2018.8310333.

12. Luschas, S., Schreier, R., \& Lee, H. S. (2004). Radio frequency digital-to-analog converter. IEEE Journal of Solid-State Circuits, 39(9), 1462-1467. https://doi.org/10.1109/JSSC.2004.829377.

13. Mayer, C., McLaurin, D., Fan, J., Bal, S., Angell, C., Gysel, O., McCormick, M., Manglani, M., Schubert, R., Reggiannini, B., Kornblum, J., Wu, L., Leonard, L., Bhal, S., Kagan, A., Montalvo, T. (2016). A direct-conversion transmitter for small-cell cellular base stations with integrated digital predistortion in 65 $\mathrm{nm}$ CMOS. In IEEE international solid-state circuits conference (pp. 63-66). https://doi.org/10.1109/RFIC.2016.7508251.

14. McLaurin, D.J., Gard, K.G., Schubert, R.P., Manglani, M.J., Zhu, H., Alldred, D., Li, Z., Bal, S.R., Fan, J., Gysel, O.E., Mayer, C.M., Montalvo, T. (2018). A highly reconfigurable $65 \mathrm{~nm}$ CMOS RF-to-bits transceiver for full-band multicarrier TDD/FDD 2G/ $3 \mathrm{G} / 4 \mathrm{G} / 5 \mathrm{G}$ macro basestations. In IEEE international solid-state 
circuits conference (pp. 162-164). https://doi.org/10.1109/ ISSCC.2018.8310234.

15. McMahill, D. R., Hurta, D. S., Brandt, B., Wu, M., Kalthoff, P., \& Ostrem, G. S. (2014). A 160 channel QAM modulator with 4.6 Gsps 14 bit DAC. IEEE Journal of Solid-State Circuits, 49(12), 2878-2890. https://doi.org/10.1109/JSSC.2014.2361352.

16. Palmers, P., \& Steyaert, M. S. J. (2010). A 10 bit 1.6-GS/s $27-\mathrm{mW}$ current-steering D/A converter with $550-\mathrm{MHz} 54-\mathrm{dB}$ SFDR bandwidth in 130-nm CMOS. IEEE Transactions on Circuits and Systems I: Regular Papers, 57(11), 2870-2879. https://doi.org/10.1109/TCSI.2010.2052491.

17. Pelgrom, M. J. M., Duinmaijer, A. C. J., \& Welbers, A. P. G. (1989). Matching properties of mos transistors. IEEE Journal of Solid-State Circuits, 24(5), 1433-1439. https://doi.org/10.1109/ JSSC.1989.572629.

18. Ravinuthula, V., Bright, W., Weaver, M., Maclean, K., Kaylor, S., Balasubramanian, S., Coulon, J., Keller, R., Nguyen, B., Dwobeng, E. (2016). A 14-bit $8.9 \mathrm{GS} / \mathrm{s}$ rf dac in 40nm $\mathrm{cmos}$ achieving $<71 \mathrm{dBc}$ LTE ACPR at $2.9 \mathrm{GHz}$. In 2016 IEEE symposium on VLSI circuits (VLSI-circuits), pp. 1-2. https://doi.org/ 10.1109/VLSIC.2016.7573538.

19. Roverato, E., Kosunen, M., Cornelissens, K., Vatti, S., Stynen, P., Bertrand, K., et al. (2017). All-digital LTE SAW-less transmitter with DSP-based programming of RX-band noise. IEEE Journal of Solid-State Circuits, 52(12), 3434-3445. https://doi.org/10. 1109/JSSC.2017.2761781.

20. Sadeghifar, M., Wikner, J. (2010). A higher Nyquist-range DAC employing sinusoidal interpolation. In NORCHIP (pp. 1-4). https://doi.org/10.1109/NORCHIP.2010.5669460.

21. Sadeghifar, M. R., Gustafsson, O., \& Wikner, J. J. (2018). Optimization problem formulation for semi-digital FIR digital-toanalog converter considering coefficients precision and analog metrics. Analog Integrated Circuits and Signal Processing,. https://doi.org/10.1007/s10470-018-1370-7.

22. Chen, Tao, \& Gielen, G. G. E. (2006). The analysis and improvement of a current-steering dacs dynamic sfdr-i: the celldependent delay differences. IEEE Transactions on Circuits and Systems I: Regular Papers, 53(1), 3-15. https://doi.org/10.1109/ TCSI.2005.854409.

23. Yang, B., Yu, Z., Lan, J., Zhang, R., Zhou, J., \& Hong, W. (2018). Digital beamforming-based massive MIMO transceiver for $5 \mathrm{G}$ millimeter-wave communications. IEEE Transactions on Microwave Theory and Techniques, 66(7), 3403-3418. https:// doi.org/10.1109/TMTT.2018.2829702.

Publisher's Note Springer Nature remains neutral with regard to jurisdictional claims in published maps and institutional affiliations.

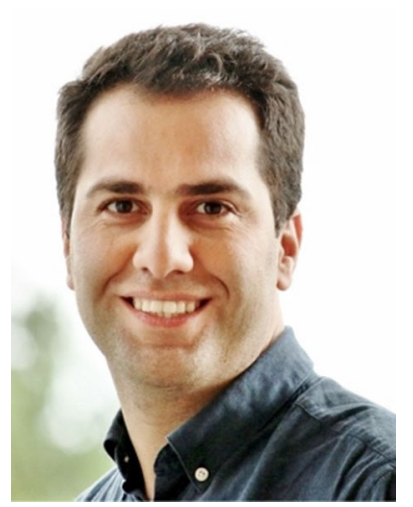

M. Reza Sadeghifar received the B.Sc. degree in electrical engineering from Beheshti University, Tehran, Iran, in 2007 and the M.S. degree in Electrical Engineering, with specialization in System-On-Chip (SoC) from Linköping University, Sweden, in 2009. He has been full-time Ph.D. researcher with Linköping University during 2010 to 2013, and since 2014 he has been part-time $\mathrm{Ph} . \mathrm{D}$. researcher with Linköping University, Sweden. Since 2014 he has also been with Ericsson AB, Radio Systems and Hardware, Stockholm,
Sweden, working on radio electronics, hardware and system design. He has served and serves as reviewer for conference ECCTD and for Journal of Analog Integrated Circuits and Signal Processing.

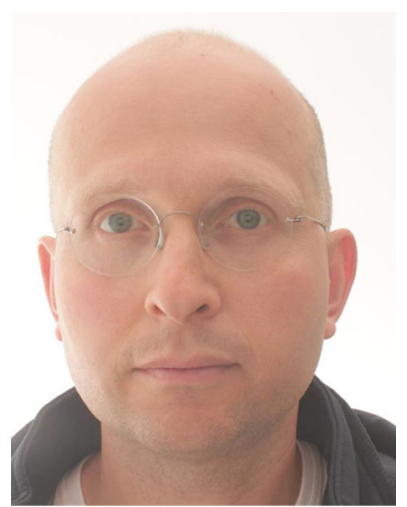

Håkan Bengtsson received the M.Sc. and Ph.D. degrees from Linköping University, Sweden, in 1999 and 2004, respectively. Between 1997 and 2011 he was working as an ultra low power RF/Analog designer at Zarlink Medical Product Group. At Linköping University he did research on a High Speed CMOS Optical receiver including decision feedback equalizers. Since 2011 he has been working as a radio base station RFIC designer at Ericsson AB.

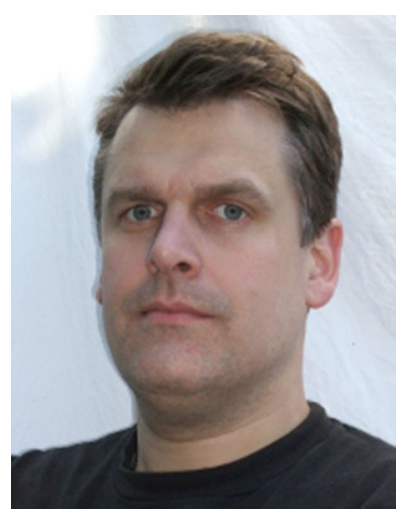

J. Jacob Wikner received his $\mathrm{Ph} . \mathrm{D}$. from the Department of Electrical Engineering, Linköping University, Sweden, in 2001. He has worked as research engineer at Ericsson Microelectronics, senior analog design engineer at Infineon Technologies, and senior design engineer and chip architect at Sicon Semiconductor. Dr. Wikner is an associate professor at Linköping University since 2009. His research interests include biologically inspired architectures, high-speed A/D and D/A converters, and general analog and mixed-signal designs. He holds six patents, has published 40 scientific papers, and has co-authored "CMOS Data Converters for Telecommunication". He is the co-founder of CogniCatus and AnaCatum Design AB. 